\title{
Privacy Vs. Daylight: Evaluating the Acceptance of Daylighting Traditional Devices in Contemporary Residential Houses in Bahrain
}

\author{
Hana M. Aljawder ${ }^{*}$, Hala A. El-Wakeel ${ }^{2}$ \\ ${ }^{1}$ Department of Architecture \& Interior Design, College of Engineering, University of Bahrain, Isa town 32038, Kingdom of \\ Bahrain \\ ${ }^{2}$ Interior Design Department, College of Design, Imam Abdulrahman Bin Faisal University, Dammam 1982, Kingdom of \\ Saudi Arabia
}

Corresponding Author Email: haljowder@uob.edu.bh

https://doi.org/10.18280/ijsdp.150508

Received: 8 January 2020

Accepted: 9 May 2020

\section{Keywords:}

acceptance, daylighting, energy, privacy, residential houses, traditional devices

\begin{abstract}
The needs and satisfaction of inhabitants with respect to daylight in Bahraini architecture is not well-established, where cultural norms significantly influence different aspects such as room division and door location. Limited consideration has been given to the needs and satisfaction of residents with respect to daylight in Bahraini architecture, where different aspects including room division and door location are influenced by cultural norms. Therefore, the study aims to assess the importance of visual privacy and daylight in the home through a survey questionnaire administered in Bahrain. The survey aimed to measure the trade-off between visual privacy and daylight at home and to explore current and traditional methods used to achieve visual privacy. Results give a clear indication of the contradictory relationship between the needed visual privacy and the desire for daylight in the space. Cultural and personal preferences are dominated by religious context. More than $95 \%$ of respondents considered visual privacy to be important; whereas, $92.07 \%$ of people considered daylight important. Moreover, $30.29 \%$ of people gave priority to the need for visual privacy over the need for daylight in the space. The visual and aesthetic aspects were clearly considered to be important factors for people.
\end{abstract}

\section{INTRODUCTION}

The visual privacy needed at home and the requirement for daylight can be mutually exclusive and be a challenge for an architect to satisfy its occupants. The need for privacy may be due to cultural, religious, personal, and psychological factors [1-4]. There is a substantial influence of spatial and architectural elements such as windows, doors, bedroom on the domestic behaviour and interactions taking place within the home environment [5]. There are strong religious traditions followed by Islam that are being applied to the structure and organization of life within the home environment. Islamic Shariah law outlines the design of traditional Muslim homes [6].

In a Muslim country, such as Bahrain, this need is generally due to a mixture of all these factors. It is important to maintain visual privacy in a Muslim community because it is considered as a sign of modesty and chastity of women since no stranger is allowed to see an unveiled woman. Cultural and personal factors also influence people's need for visual privacy in Bahrain. As a result, people have developed a tendency to cover their windows, which affects the level of daylight in their homes. The factor of visual privacy remains a dilemma among the Muslim community. Moreover, other alternatives, such as; mashrabiya/rawshan have also been abhorred by the Muslim natives [7]. However, the people of Bahrain exhibit a strong desire for visual privacy, which creates challenges for architects. Moreover, the reduction of daylight can lead to more energy consumption, which can be mitigated during the day by consuming more daylight for regular activities [8]. Furthermore, associated health benefits with daylight can also be reduced due to concerned visual privacy.

Recently, previous studies have proposed the use of light pipes, remote-source lighting systems, optical units, and light shelves for bringing daylight into rooms more efficiently and intentionally as well as enhancing privacy and comfort. People's attention has been aroused through increased consciousness regarding privacy to their living conditions such as luminous comfort, acoustic comfort, and thermal comfort. Arifin et al. [9] have investigated the daylight performance of residential buildings in Malaysia and reported their physical environments by examining five important constructs that influence interior daylight levels such as window area, shading, external obstruction, building area and orientation, and glass type. Lau et al. [10] have observed residential preferences for solar access in high-density topical cities in Hong Kong.

Daylight is a core architectural aspect in the current study because the alternative to daylight is the dependence on artificial lighting, which can reveal a significant incline in energy consumption and contribute to the issue of global warming [11]. In Middle Eastern countries, intensive heat entering houses from contemporary building design leads individuals for consuming energy using air-conditioning systems [12]. The ratio of windows to different interior surfaces such as the floor or wall for finding the best 
geometrical ratio between interior area and windows regarding window to interior walls ratio or window to floor area ratio. The application of Western design concepts has been criticized by Arabian architects as driving to a loss of local identity in the built environment [13]. Rawshan provides shading from direct sun and general climatic modification in the traditional architecture of Middle Eastern countries. Changes in meaning and utility are related to the diminished use of Rawshan.

Several forms of lighting are essential, but it is not identified from the perception of a house inhabiting a building. Bahraini architects have criticized the application of Western design notions in Bahrain, which leads to a loss of local identity in the housing environment. Mashrabiya is an architectural element that offers daylight, privacy, and ventilation as well as offers shading from direct sun and climatic changes, at the same time. It also provides a set of important design considerations in the conventional architecture of Bahrain. It has been observed that the reduced use of Mashrabiya in Bahrain is linked with modifications in utility and meaning. A recent study on Bahrain emphasized the importance of mashrabiya in Islamic architecture, specifically in Bahrain for reducing the glare from direct sunlight, providing visual privacy, and allowing natural ventilation. Furthermore, the study indicated that the design of Bahraini houses is based on particular characteristics that effectively address the religious and cultural needs of the bio-climatic conditions and residents. Similarly, the study strongly revealed that uniformity of daylight in space is an important feature of the mashrabiya device that reduce the impact of glare in the whole space [14].

Limited consideration has been given to the needs and satisfaction of inhabitants with respect to daylight in Bahraini architecture, where cultural norms significantly influence different aspects such as room division and door location. Thereby, this study fills this gap by examining architectural design in contemporary houses in Bahrain with respect to interior daylight levels. Muslims are exposed to different cultures and traditions of their host countries as they migrate. The ways in which Muslims achieve and perceive privacy and extend hospitality within their homes have been discussed previously. However, studies discussing the association of privacy settings and exposure of Muslims to daylight are lacking. In a similar context, the present study aims to evaluate the relationship between the power of privacy and daylight among the residents of Bahrain. The study aims to determine people's satisfaction with the visual privacy of their homes. Moreover, it determines the trade-offs between visual privacy and daylighting for Bahraini people. Furthermore, the study aims to explore the opinions of the people of Bahrain towards the following:

- The acceptance of approaches and methods used in houses to achieve visual privacy.

- The acceptance of traditional approaches used in houses to achieve visual privacy (e.g., the mashrabiya).

- The reasons behind people's reluctance to use traditional methods, such as mashrabiya, at their homes.

- The acceptance of a revamped mashrabiya concept for use in homes to achieve visual privacy.

The study proves to be significant for the architects in developing methods and strategies to overcome the existing issues of privacy among the Muslim community. Moreover, the study provides sufficient knowledge on the required topic which proves to be significant for future work in the desired field. Furthermore, the choices and preferences of the Bahraini people are also evaluated to predict the direction of future work. The use of daylight is an important aspect of sustainable buildings as it is observed for reducing the use of electricity. On the contrary, the extensive incidence of multi-occupied spaces inhibiting the use of manual controls, the eradicated power density of modern electric lighting systems, and the limitations of automatic lighting control systems practically, have made it complicated for justifying the cost of daylighting based on the potential energy savings. It is essential for explaining that the widespread use of daylighting in buildings has fundamental effects in other areas that have a financial effect on occupying the building and the organization owns.

\section{LITERATURE REVIEW}

The architectural identity of cities is affected by a different reason, which results from the appearance of new movements and styles in defining new cultural personas. The local architects educated in Western Countries import urban design practices to widen current streets and different parts of the conventional cities [15]. Furthermore, the widespread use of concrete, glass, and steel represents innovative building technologies and materials, which considered signs of development and progress.

The physical environments of buildings are usually explained simply and such aspects are usually failed to consider individual preferences for lighting conditions. Human behaviour is evaluated for taking better consideration of lighting factors with respect to lighting. Williams et al. [16] have considered the architectural design from the perspectives of nature-based activities of residents and from a home. Nature plays a very important role to shape preferences and their sense of wellbeing of people. Weidemann et al. [17] have developed a theoretical framework to measure indoor productivity for evaluating the behavioural environment. The study has shown that behaviour and structure influenced significantly on productivity. Van Esch et al. [18] have included gender, age, seasonality of mood for modifying a model related to psychological and physical discomfort.

Privacy is defined as the condition of being protected from unwanted access by personal information, attention, and physical access. Privacy is accessible in different cultures and societies with variations [19]. Women are needed to have privacy from being observed by men who are not Mahram since Islam is the religion of all Muslims and it influences most areas of their lives. Hashim and Rahim [20] have defined privacy as a two-way process that involves the absorptivity of boundaries between others and oneself. Public privacy is the privacy between residents inside their homes and individuals outside the home. Similarly, private privacy is the privacy between family members. Opening and closing barriers can provide privacy in architecture and reveal as a major aspect of designers' and architects' jobs. The concept of privacy is associated with human needs, but the specification of such needs differs between cultures [21]. In architecture design, this individual private space is needed in most outdoor and indoor areas. For instance, the decrease in privacy levels results in confidentiality at work and staff satisfaction in an office building. Kim and Dear [22] have reported that open-plan offices result in staff dissatisfaction due to the lack of individual privacy, even though they are considered places that help with staff interaction and cooperation. 
Home is considered as a place that offers residents with cosiness, wellbeing, privacy, and comfort. Al-Thahab et al. [23] have mentioned that the concept of privacy links to the association between public and private spaces throughout homes. The importance of privacy between genders at home in the Arabian Gulf region regarding room separation between men and women in reception zones is essential. The absence of privacy in Montreal residences is one of the biggest issues that influence the comfort of residents, who are Arab Muslims.

Aljawder et al. [14] mentioned that mashrabiya is the device used in traditional Islamic architecture to provide visual privacy between public and private spaces as well as to reduce the glare from direct sunlight, and allow natural ventilation. Aljawder further claimed that privacy and bio-climatic comfort are perhaps the most important effects on traditional Bahraini houses.

Daylighting is an essential parameter of building comfort. Traditional houses typically take benefit of natural light due to restricted building and technical resources available at the time they were created [24]. On the contrary, original daylighting circumstances are not competent for meeting the demands of the present with modifications in living habits as well as the enhancement of living standards. Enhancements of vernacular houses must also emphasize to improve daylighting circumstance, an area which requires to be evidently reported comprehensively. Previously, few studies have been conducted on architectural daylighting of traditional daylighting buildings, but it is a generally neglected area of research to be concentrated on [25-27].

A strong association between gender and architecture in vernacular homes has been shown in the literature review. In particular, the identity of women is considered in conventional architecture, but previous studies have allowed exposing an existing gap in this area [28]. Thereby, the influence of poor daylight in contemporary houses on daily lives of women and wellbeing should be examined. Factors behind cultural and religion barriers should be explored even though a lack of daylight will influence individuals in general [13]. Impairment can be considered a disruptive factor, which may influence their daily lives and wellbeing when women cannot solve the issue to acquire enough daylight at home.

\section{METHODS}

\subsection{Research population}

The study randomly selected people living in Bahrain (Bahraini and non-Bahraini citizens) to evaluate different requirements for visual privacy and daylight inside their houses. The targeted population in this survey included people of both genders aged 18 years or older.

\subsection{Sample size}

The total population of Bahrain, according to the latest statistical study published by CIO is $1,635,722$ (Bahraini and non-Bahraini citizens). The target population in this research from Figure 1 was 986,968 (Table 1). The suitable sample size for the research that would represent the whole community was determined using (Eq. (1)) from the National Education Association [29].

$$
s=\frac{X^{2} N P(1-P)}{d^{2}(N-1)+X^{2} P(1-P)}
$$

where,

$\mathrm{s}=$ required sample size

$\mathrm{X}=$ the value of the chi-square for one degree of freedom $=$

(3.841) at a confidence level of $(0.05)$

$\mathrm{N}=$ the population size

$\mathrm{P}=$ the population proportion

$\mathrm{d}=$ the degree of accuracy expressed as a proportion $(0.05)$

Table 1. The required sample size according to the National Education Association

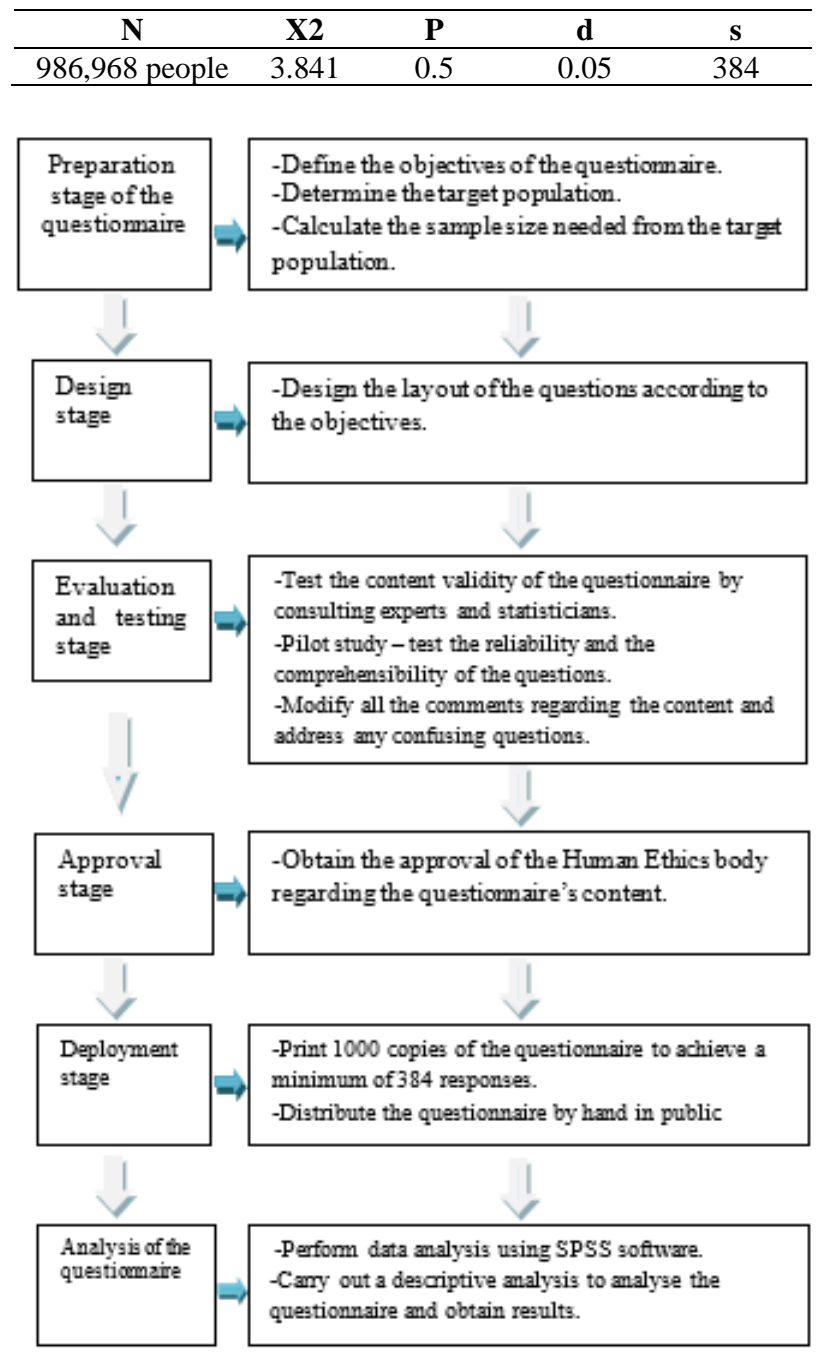

Figure 1. The stages of the questionnaire design

\subsection{Instrument}

A descriptive, cross-sectional survey close-ended questionnaire was used as the study instrument that was designed to measure several issues related to visual privacy and daylight in Bahraini homes. The rationale behind using this method was its convenience to obtain a large number of responses from a population. It can provide information regarding people's opinions and behaviour regarding specific issues through particular questions. The questionnaire comprised of four sections, with each section aiming to address one or more of the aforementioned objectives. These four sections included 15 questions. The first section included questions to evaluate the existing levels of visual privacy and to assess its importance in Bahraini homes. Moreover, the study aims to examine the interaction between privacy and the provision of daylight inside homes. The second section 
included questions to probe the extent of occupants' acceptance of different approaches used to achieve visual privacy in their homes and their preferences regarding these approaches. The third section includes questions to evaluate the willingness or reluctance of Bahraini residents to use traditional methods to achieve visual privacy, as well as reasons underlying their attitudes toward these methods. The last section of the questionnaire includes questions to explore occupants' acceptance of innovation in older approaches and their attitudes toward using them in their houses. A five-point Likert scale (very satisfied - very dissatisfied or very important - not important) was used to obtain reliable responses. The research was conducted in several stages, as shown in Figure 1.

\subsection{Validity and reliability}

In this study, the aim was to address face and content validity. The validity face was selected to ensure that the instrument was easy to use, clear and readable while content validity was assessed to ensure that the questions were accurate, credible and relevant to the field of study [30-32]. The draft questionnaire was presented to two experts: one in the field of architecture and one in the field of lighting. This procedure was carried out to ensure that the questions were related to and would measure the objectives of the study.

Reliability refers to the reproducibility and consistency of a questionnaire or an instrument, which means that the questionnaire must consistently measure what it is intended to measure. Several types of reliability tests can be applied, such as test-retest reliability, parallel-forms reliability, internal consistency, split-half, Cronbach's Alpha $(\alpha)$, etc. Each depends on the type of data to be gathered and whether these are nominal, ordinal or interval/ratio in scale. In this study, test-retest reliability and Cronbach's Alpha $(\alpha)$ were the most appropriate methods as the questionnaire instrument produced nominal and ordinal data. The test-retest reliability method is based on asking people to complete a self-administered questionnaire twice on separate occasions, with two to three weeks in between. Moreover, two responses can be statistically compared using Spearman's rank correlation coefficient, Pearson's correlation coefficient or Kendall's correlation coefficient [33]. Testing the reliability of the questionnaire showed that Cronbach's Alpha $(\alpha)$ for the whole sample size was 0.719 , which indicates an acceptable level of reliability since an acceptable level range between $0.7-1$. The validity of an instrument includes four tests: face, content, criterion, and construct.

In the pilot study, reliability was checked using the testretest method. This was achieved by distributing the questionnaire to a sample of 20 people and then, after two weeks, the questionnaire was redistributed for a second time to the same sample. The Kendall correlation coefficient was calculated using SPSS version 20 to check the consensus between the two sets of responses. The value of the correlation coefficient of the total score of the scale was 0.764 ; this value is statistically significant at a 0.01 level of significance. Table 2 shows the compatibility coefficients of all the instrument's statements in pre and post applications. From Table 2, it can be seen that the correlation coefficient between the two applications of all the statements ranged from 0.427-1. The majority of the correlation coefficients were statistically significant at 0.01 , which indicates the presence of high consensus in the responses of the respondents in the two applications. This shows that the instrument demonstrated a high level of consistency.

Table 2. The compatibility coefficients of all the instrument's statements in pre and post applications

\begin{tabular}{|c|c|c|c|c|c|c|c|}
\hline Item & $\begin{array}{c}\text { Correlation } \\
\text { coefficient }\end{array}$ & Item & $\begin{array}{c}\text { Correlation } \\
\text { coefficient }\end{array}$ & Item & $\begin{array}{c}\text { Correlation } \\
\text { coefficient }\end{array}$ & Item & $\begin{array}{c}\text { Correlation } \\
\text { coefficient }\end{array}$ \\
\hline 1 & $0.490 *$ & 6 & $0.575^{* *}$ & $9 a$ & $1.00 * *$ & 10 & $1.00^{* *}$ \\
\hline $2 \mathrm{a}$ & $0.688 * *$ & $7.1 \mathrm{a}$ & $0.850 * *$ & $9 \mathrm{~b}$ & $0.892 * *$ & 11 & $.744 * *$ \\
\hline $2 \mathrm{~b}$ & $1.00 * *$ & $7.1 \mathrm{~b}$ & $0.895 * *$ & $9 \mathrm{c}$ & $0.793 * *$ & 12 & $.901 * *$ \\
\hline $2 \mathrm{c}$ & $0.596 * *$ & $7.1 \mathrm{c}$ & $0.633 * *$ & $9 \mathrm{~d}$ & $0.707 * *$ & 13 & $1.00 * *$ \\
\hline $2 \mathrm{~d}$ & $1.00 * *$ & $7.2 \mathrm{a}$ & $0.616 * *$ & $9 \mathrm{e}$ & $0.899 * *$ & 14 & $1.00 * *$ \\
\hline $2 \mathrm{e}$ & $1.00 * *$ & $7.2 \mathrm{~b}$ & $0.669 * *$ & $9 f$ & $0.729 * *$ & $15 \mathrm{a}$ & $1.00 * *$ \\
\hline 3 & $0.427 *$ & $7.3 \mathrm{a}$ & $0.800 * *$ & $9 j$ & $1.00 * *$ & $15 b$ & $0.533^{*}$ \\
\hline 4 & $1.00 * *$ & $7.3 \mathrm{~b}$ & $0.731 * *$ & $9 \mathrm{~h}$ & $1.00 * *$ & $15 \mathrm{c}$ & $.882 * *$ \\
\hline 5 & $0.772 * *$ & 8 & $0.690 * *$ & $9 \mathrm{i}$ & $1.00 * *$ & $15 \mathrm{~d}$ & $1.00 * *$ \\
\hline
\end{tabular}

* Correlation is significant at the 0.05 level; ** Correlation is significant at the 0.01 level

\subsection{Data analysis}

SPSS v20 was used to calculate the percentages and frequencies to help compare the responses for each question and thus, evaluate people's preferences. A chi-square test and correlation coefficient were applied to compare the significance of the proportions of some of the qualitative response variables at $\mathrm{P}$-value $\leq 0.05[34,35]$.

\section{RESULTS}

Among 416 respondents living in Bahrain, 138 (33.2\%) were men and $278(66.8 \%)$ were women; thus, the men to women ratio was $1: 2$. A total of $349(83.9 \%)$ of the respondents were Bahraini and $76(16.1 \%)$ belonged to a different nationality. In terms of religion, 403 (96.9\%) of the respondents were Muslims, $8(1.9 \%)$ were Christian and 5 $(1.2 \%)$ were from other religions. To answer three questions mentioned earlier, the study analysis was divided into questions as follows:

\subsection{Occupant preferences for visual privacy and daylight in homes}

The first section of the questionnaire included six questions to gain information about the level of visual privacy that the occupants desired in their houses. The methods used to maintain this visual privacy, and the desired and achieved a level of daylight at home were also evaluated. Regarding the importance of visual privacy, a significantly high proportion of respondents $(95.91 \%)$ considered it to be important. 
Regarding achieving visual privacy in their houses, the responses showed that 343 people $(82.45 \%)$ maintained visual privacy by lowering a curtain, 149 people (35.82\%) used reflective glass, 101 people $(24.28 \%)$ were using high fences, and 88 people $(21.15 \%)$ used plants and trees to maintain their visual privacy. The findings illustrated in Figure 2 show that maintaining visual privacy in the home was very important for people; while, satisfaction with the level of visual privacy achieved was also very high.

A total of $92.07 \%$ of respondents confirmed the importance of daylight in their houses. Very few responses were obtained that indicated daylight to be of moderate or less importance. In response to a question about their satisfaction with the level of daylight in their houses, $76.44 \%$ of respondents were satisfied, $12.50 \%$ said neither satisfied nor dissatisfied, and $11.06 \%$ of people replied with dissatisfied and very dissatisfied. Table 3 indicates that $49.28 \%$ of respondents reported the importance of both visual privacy and daylight.

It is clear from this result that the majority of respondents did not differentiate between the importance of visual privacy and daylight as they considered them to be equally important. This was confirmed by respondents' answers to the sixth question of the questionnaire. In addition, the results of the chi-square test showed that differences occurring between the percentages were statistically significant, as the chi-square equaled 34.41; this is statistically significant at $\mathrm{P}$-value $<0.01$. Furthermore, the correlation coefficient (phi) was calculated to indicate people's responses towards these two questions; which is statistically significant at $\mathrm{P}$-value $<0.01$.

In the sixth question, the questionnaire aimed to evaluate the trade-off between daylight and visual privacy for Bahraini people (Figure 3). Earlier responses to questions one and four indicated that $95.91 \%$ and $92.07 \%$ of people confirmed the importance of visual privacy and daylight, respectively. $12.02 \%$ of people found both to be important indicating that the level of daylight was more important. $11.78 \%$ of the sample prioritized admitting daylight into space. However, $6.49 \%$ of the sample said that visual privacy was the most important to them.

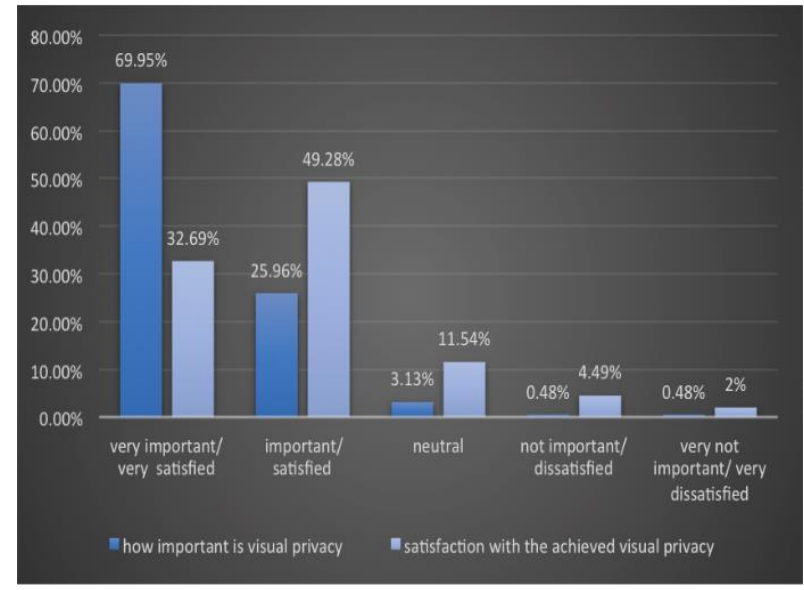

Figure 2. Comparison between the importance of visual privacy and the level of satisfaction with the visual privacy achieved

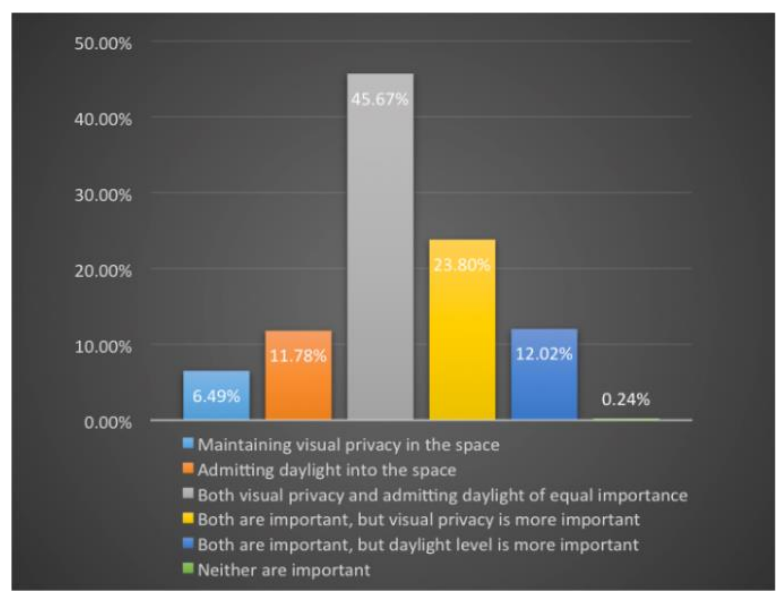

Figure 3. The trade-off between daylight and visual privacy for Bahraini people

Table 3. A contingency table indicating the relationship between the importance of visual privacy and daylight

\begin{tabular}{ccccccccccc}
\hline Daylight Level & \multicolumn{2}{c}{ Very important } & \multicolumn{2}{c}{ Important } & \multicolumn{2}{c}{ Moderate } & \multicolumn{2}{c}{ Less important } & \multicolumn{2}{c}{ Not important } \\
\cline { 2 - 11 } = Visual Privacy & $\mathrm{F}$ & $\%$ & $\mathrm{~F}$ & $\%$ & $\mathrm{~F}$ & $\%$ & $\mathrm{~F}$ & $\%$ & $\mathrm{~F}$ & $\%$ \\
\hline Very important & 205 & $49.28 \%$ & 67 & $16.11 \%$ & 15 & $3.61 \%$ & 4 & $0.96 \%$ & 0 & $0.00 \%$ \\
Important & 50 & $12.02 \%$ & 46 & $11.06 \%$ & 12 & $2.88 \%$ & 0 & $0.00 \%$ & 0 & $0.00 \%$ \\
Moderate & 3 & $0.72 \%$ & 8 & $1.92 \%$ & 2 & $0.48 \%$ & 0 & $0.00 \%$ & 0 & $0.00 \%$ \\
Less important & 2 & $0.48 \%$ & 0 & $0.00 \%$ & 0 & $0 \%$ & 0 & $0.00 \%$ & 0 & $0.00 \%$ \\
Not important & 1 & $0.24 \%$ & 1 & $0.24 \%$ & 0 & $0 \%$ & 0 & $0.00 \%$ & 0 & $0.00 \%$ \\
\hline
\end{tabular}

\subsection{Occupants' acceptance of and preference for visual privacy techniques used in window design}

The questions aimed to evaluate people's acceptance of different techniques used to maintain visual privacy in terms of window design. The methods and techniques used in window design were classified into three categories to achieve visual privacy:

- Physical obstruction techniques: These obstruct the image of the occupants (e.g., mashrabiya and shutters).

- Optical manipulation techniques: It distorts the image so that figures are unrecognizable (e.g., frosted glass and textured glass).
- Optical transmittance techniques: The technique reduces the transmittance of the image (e.g., coloured glass and reflected glass).

The techniques were arranged in the following order in terms of preference: 334 people $(80.29 \%)$ said showed interest reflective glass; whereas, 276 people $(66.35 \%)$ showed interest in moveable shutters (Figure 4). Similarly, $46.16 \%$ and $44.23 \%$ were interested in textured glass and automated mashrabiya, respectively (Figure 4). Moreover, $43.03 \%$ were interested in frosted glasses. The last two techniques were coloured glass and the fixed mashrabiya in order of people's preference. It has been stated that $32.93 \%$ of people were interested in the coloured glass and $30.53 \%$ of people were very interested and interested in the fixed mashrabiya (Figure 4). 


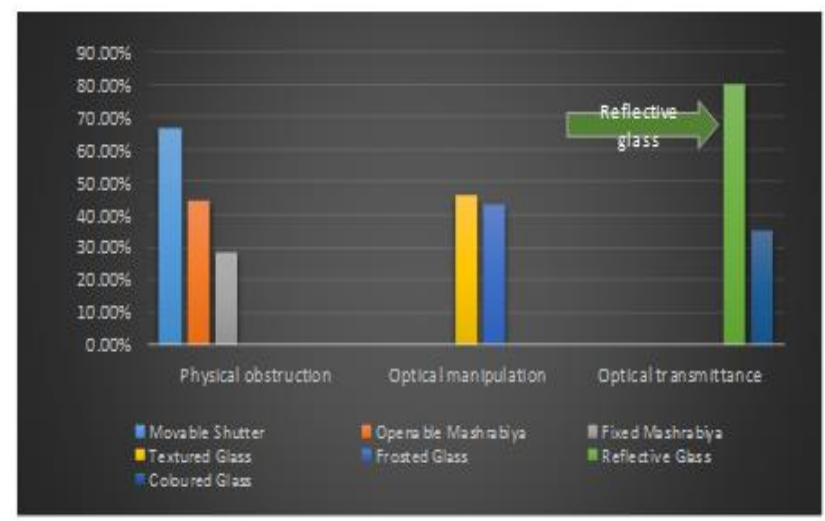

Figure 4. People's acceptance of the different techniques used to maintain visual privacy

\subsection{Occupants' interest in using traditional methods (the mashrabiya) to achieve visual privacy in their homes}

This section aimed to explore whether or not people were interested in using traditional Mashrabiya. Secondly, the reasons behind their reluctance to use the traditional mashrabiya, and their opinion of the mashrabiya performance in maintaining the desired visual privacy at home. Lastly, whether the daylight level provided by mashrabiya could or could not offer visual comfort to the occupants. The results indicate that the use of the traditional mashrabiya is not a priority for people who want visual privacy as $40.38 \%$ of people were interested $(12.74 \%$ of people were very interested and $27.64 \%$ of people were interested), $33.42 \%$ were uninterested, and $23.08 \%$ of people were neutral toward the use of this technique.

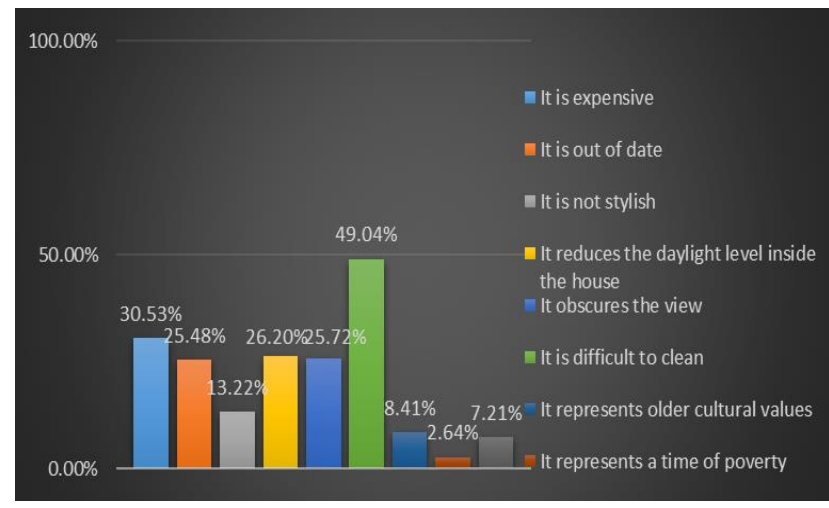

Figure 5. The percentages of responses for reasons behind not using the mashrabiya

Figure 5 shows that $49.04 \%$ of the responses are not using traditional Mashrabiya, followed by the reason that (it is expensive) with $30.53 \%$ of the responses. $26.20 \%$ of the people gave the reason that (it reduces the daylight level inside the house). Moreover, (it obscures the view) and (it is out of date) had almost the same percentages of $25.72 \%$ and $25.48 \%$ of responses, respectively. It has also been shown that $13.22 \%$ and $8.41 \%$ of respondents responded with "not stylish" and "represent older cultural values", respectively.

The questionnaire included whether any of the respondents had experienced being in a place with a Mashrabiya, to test whether the above responses were the result of hypothetical opinions or due to experience. From 416 respondents, 217 $(52.16 \%)$ of them had experienced it being in a place covered by a Mashrabiya. 169 people (77.88\%) agreed that the Mashrabiya can provide the visual privacy needed by occupants $(52.53 \%), 16.59 \%$ of people were neutral, and only $5.53 \%$ of people disagreed. Furthermore, with regard to the ability of the mashrabiya to provide visual comfort concerning the level of daylight for the occupants, $46.55 \%$ of the 217 respondents agreed with this statement, $28.57 \%$ of people were neutral, and $24.88 \%$ of people disagreed with the statement.

\subsection{Receptivity towards using innovations of the mashrabiya}

In this section of the questionnaire, the people's acceptance of the new mashrabiya designed using various new techniques was measured. Respondents were asked about the types of façade covering and materials that could be used for the new mashrabiya, after offering some images of the new mashrabiya design with different materials and different coverings (Figure $6)$.

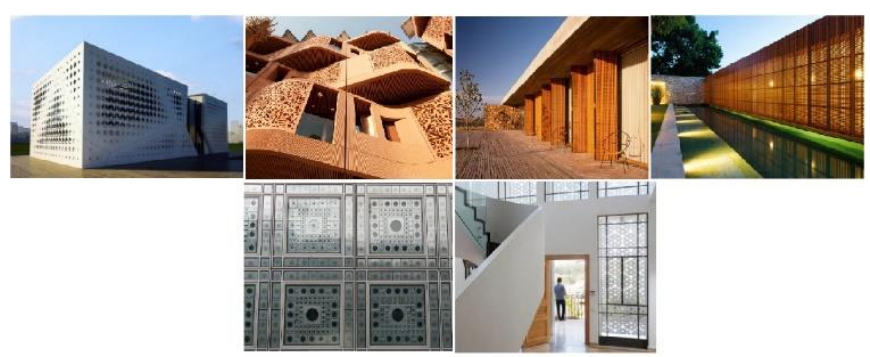

Figure 6. Images of the innovative mashrabiya designs illustrated in the questionnaire

These methods provided cover in two different ways:

- A facade cover which covered the entire facade of the building.

- A window opening covers covering the window opening.

Both types were presented as fixed or movable. Additionally, newer versions of mashrabiya were introduced using materials other than wood, such as concrete, aluminium and steel covered with glass. When the participants were asked, if the mashrabiya were redesigned in a modern way, what type of cover they would prefer, $36.06 \%$ preferred to cover the window opening only; while $35.34 \%$ preferred a mixture of both covering (window and the entire façade). However, $22.60 \%$ still preferred not to use mashrabiya at all. Furthermore, when the participants were questioned about the mashrabiya ability to open, people mostly preferred (51.44\%) to use an openable mashrabiya.

Wooden mashrabiya covered with glass was indicated as the first choice by $42.55 \%$ of the study sample, followed by steel or aluminium mashrabiya covered with glass as the second choice by $32.45 \%$ of the respondents; while, concrete mashrabiya covered with glass as the third option. Referring to the previous section, $139(33.42 \%)$ respondents were either uninterested or very uninterested when they were asked about using the mashrabiya to achieve visual privacy in their homes. However, 93 from the same 139 respondents changed their minds by indicating the method and material they would prefer to use for the new design of mashrabiya, when they were introduced to some images of the new mashrabiya designs. 


\section{DISCUSSION}

The importance of daylight for the residents of Bahrain stems from their belief of its importance, partly because Bahrain is a sunny country throughout the year and also old Bahrainis used to live in courtyard houses where they spent most of the day. Thus, many of the respondents used reflective glass or high fence/plants to maintain their visual privacy as well as daylight in their houses. On the other hand, 42 people $(91.3 \%)$ out of 46 indicated that the level of daylight was very important to them; although, just a small proportion of people (46-11.06\%) were not satisfied with the achieved daylight level in their homes. This might be because of the influence of the method used to achieve visual privacy in their homes.

The people's focus of interest has changed and moved towards the currently available architectural and building technology. This is again because of social and economic changes, and globalization. People want new building technology as it allows them to feel that their home is contemporary and up-to-date. Using reflective glass over a building's façade can give people this feeling as represented by $80.29 \%$ of people who are interested in reflective glass.

Only $40.38 \%$ of respondents were interested in using the traditional type of mashrabiya, while the others were not interested in it. The reasons behind this reluctance could be classified as both personal and cultural. The personal reasons relied on peoples' tastes and interests in terms of a building's appearance. For example, it reduces the level of daylight inside the house, it obscures the view and enhances the aesthetic of the building, it is out of date, or it is not stylish. These reasons also relied on affordability: for example, some respondents said it is expensive and difficult to clean. Whereas cultural reasons include, older cultural values and that it represents a time of poverty.

Therefore, it may be concluded that the main reasons for not using traditional devices such as the mashrabiya are aesthetics. This means that the other reasons mentioned earlier (such as it is difficult to clean, it is expensive and it reduces the daylight) might not really affect the acceptability of a new design if it is produced according to their tastes and preferences. The findings suggested that people mainly prefer a window cover or a mixture of both a window and a wall cover in the case of renovating the traditional mashrabiya. The mashrabiya must be openable and will most likely be made of wood covered with glass.

The identity should be preserved and the repetition of theoretical discussion regarding Mashrabiya should be avoided. This allows architects to make the Mashrabiya more unique with the most adequate safety and cost and for maintaining the western architecture renaissance. An integrated trend is represented by the contemporary interpretation pattern, which instigates a successful approach for the combination of modern technologies and traditional Arabic building practices. This pattern takes the advantages of the latest progressions in producing a sustainable design and inhibits them throughout the heritable aspect's framework. It is essential for indicating that the idea of contemporary mashrabiya is an adequate solution in the Arab cities for highrise residential buildings where a high-density building is required.

The study contributes to knowledge in identifying that issues with psychological and physical privacy should be discussed from different perspectives, not only from the health perspective, as a result of poor daylight exposure. It is essential to discuss this matter from an architectural perspective. This is specifically the case in particular cultures and regions such as Bahrain, where residents stay inside buildings due to heat and privacy. This study contributes to the existing studies regarding domestic and daylight environments to clarify the essence of privacy from the perspectives of residents and how their perspectives are not considered. This study will help municipalities and other authorities in controlling building regulations by clarifying the physical and psychological effects on residents. This study might affect policy-makers for enhancing regulations and offer strict regulations that assure penetration of appropriate daylight inside flats and living rooms.

\section{CONCLUSION}

The study has assessed the importance of visual privacy and daylight in the home through a survey questionnaire administered in Bahrain. The contradictory answers showed that religious belief is the dominant reason for the need for visual privacy; although, the need for visual privacy derives from different reasons, such as religious beliefs, personal preferences, and cultural factors. The findings of the current study suggest that culture and personal preferences are influenced by the religious context. This is exemplified by the fact that some people did not perceive visual privacy as a priority and they respected and adhered to the religious society they lived to a certain extent. People were aware of the conflict between the need for visual privacy and the availability of daylight in their houses; therefore, reflective glass, which had a high percentage of acceptance, was the most appropriate solution for many as it can provide the visual privacy needed during the daytime while admitting daylight into space. The results also showed that people were willing to accept the idea of renovating the traditional method if certain guidelines are followed.

In addition, the influence of the desire to admit daylight into space can be seen as they still want to remain in contact with the outside world, despite people's need for visual privacy. Thus, reflective glass was preferred by most of the respondents, as this ensures that people can enjoy the daylight and connect with the world outside at the same time while maintaining visual privacy. Moveable shutters also give the impression that people need to be free to control both their visual privacy and the daylight that enters the space. Therefore, people priorities the first and third techniques (physical obstruction techniques and optical transmittance techniques) that can provide both visual privacy and daylight.

\section{ACKNOWLEDGEMENTS}

The authors are very thankful to all the associated personnel in any reference that contributed in/for the purpose of this research. Further, this research holds no conflict of interest and is not funded through any source.

\section{REFERENCES}

[1] Altman, I. (1975). The Environment and Social Behaviour: Privacy, Personal Space, Territory, and Crowding. Cole Publishing Company, Monterey, 
California.

[2] Newell, P.B. (1995). Perspectives on privacy. Journal of Environmental Psychology, 15(2): 87-104. https://doi.org/10.1016/0272-4944(95)90018-7

[3] Al-Kodmany, K. (1999). Residential visual privacy: Traditional and modern architecture and urban design. Journal of Urban Design, 4(3): 283-311. https://doi.org/10.1080/13574809908724452

[4] Smith, J.M. (2001). Personal privacy: Cultural concerns International Encyclopedia of the Social \& Behavioral Sciences, 2001: 11250-11254. https://doi.org/10.1016/b0-08-043076-7/04623-4

[5] Heathcote, E. (2012). The Meaning of Home (Illustrated ed.). Frances Lincoln Limited, London.

[6] Othman, Z., Aird, R., Buys, L. (2015). Privacy, modesty, hospitality, and the design of Muslim homes: A literature review. Frontiers of Architectural Research, 4(1): 12-23. https://doi.org/10.1016/j.foar.2014.12.001

[7] Aljawder, H.M.H. (2014). Residential window: Daylight vs. visual privacy. PhD dissertation, School of Architecture, Design and Planning, University of Sydney, Sydney, Australia.

[8] Husin, S., Fairuz, S.N., Hanur Harith, Z.Y. (2012). The performance of daylight through various windows for residential buildings/Sharifah Nor Fairuz Syed Husin and Zarina Yasmin Hanur Harith. Asian Journal of Environment-Behaviour Studies, 3(8): 85-96. https://doi.org/10.21834/aje-bs.v3i6.247

[9] Arifin, N.A., Abdullah, Z., Yeap, K.S. (2017). Parametric studies on building separation of daylight performance in obstructed low-cost high-rise residential building through computer simulation techniques in Kuala Lumpur, Malaysia. Science International, 29(1): 93-93.

[10] Lau, K.L., Ng, E., He, Z.J. (2011). Residents' preference of solar access in high-density sub-tropical cities. Solar Energy, 85(9): $1878-1890$ https://doi.org/10.1016/j.solener.2011.04.026

[11] Gago, E.J., Muneer, T., Knez, M., Köster, H. (2015). Natural light controls and guides in buildings. Energy saving for electrical lighting, reduction of cooling load. Renewable and Sustainable Energy Reviews, 41: 1-13. https://doi.org/10.1016/j.rser.2014.08.002

[12] Assali, I.M. (2017). Buildings façades a challenge of cultural identity in the context of modernity. IOSR Journal of Environmental Science, Toxicology and Food Technology, 11(3): 98-107. https://doi.org/10.9790/2402-11030298107

[13] Shatwan, A.M. (2018). Domestic window design and interior daylight in Jeddah: Designing for Saudi women. $\mathrm{PhD}$ Theses, University of Hertfordshire. https://doi.org/10.18745/th.20278

[14] Aljawder, H.M., El-Wakeel, H.A. (2019). Evaluating the performance of a daylighting traditional device, The mashrabiya, in clear sky conditions: Case study of a traditional Bahraini house. WIT Transaction on The Built Environment, 191: 395-409. https://doi.org/10.2495/STR190341

[15] Rashid, M., Ara, D. (2020). Tectonics in the gulf architecture: 'Modernity of tradition' in buildings. Building in Hot and Humid Regions, pp. 137-150. https://doi.org/10.1007/978-981-13-7519-4_6

[16] Williams, K.J., Lee, K.E., Sargent, L., Johnson, K.A., Rayner, J., Farrell, C., Williams, N.S. (2019). Appraising the psychological benefits of green roofs for city residents and workers. Urban forestry \& Urban Greening, 44: 126399. https://doi.org/10.1016/j.ufug.2019.126399

[17] Francescato, G., Weidemann, S., Anderson, J.R. (2018). Evaluating the built environment from the users' perspective: Implications of attitudinal models of satisfaction. In Building Performance Evaluation, Springer, Cham, 87-97. https://doi.org/10.1007/978-14899-3722-3_14

[18] Van Esch, E., Minjock, R., Colarelli, S.M., Hirsch, S. (2019). Office window views: View features trump nature in predicting employee well-being. Journal of Environmental Psychology, 64: 56-64. https://doi.org/10.1016/j.jenvp.2019.05.006

[19] Fakere, A.A., Arayela, O., Folorunso, C.O. (2017). Nexus between the participation of residents in house design and residential satisfaction in Akure, Nigeria. Frontiers of Architectural Research, 6(2): 137-148. https://doi.org/10.1016/j.foar.2017.02.003

[20] Hashim, A.H., Rahim, Z.A. (2010). Privacy and housing modifications among Malay urban dwellers in Selangor. Pertanika Journal of Social Science and Humanities, 18(2): 259-269.

[21] Węziak-Białowolska, D., Dong, Z., McNeely, E. (2018). Turning the mirror on the architects: A study of the openplan office and work behaviors at an architectural company. Frontiers in Psychology, 9. https://doi.org/10.3389/fpsyg.2018.02178

[22] Kim, J., De Dear, R. (2013). Workspace satisfaction: The privacy-communication trade-off in open-plan offices. Journal of Environmental Psychology, 36: 18-26. https://doi.org/10.1016/j.jenvp.2013.06.007

[23] Al-Thahab, A., Mushatat, S., Abdelmonem, M.G. (2014) Between tradition and modernity: Determining spatial systems of privacy in the domestic architecture of contemporary Iraq. International Journal of Architectural Research, $\quad 8(3)$ : 238-250. https://doi.org/10.26687/archnet-ijar.v8i3.396

[24] Xuan, H., Wu, P.C., Su, D.W. (2013). Daylighting analysis of vernacular architecture in Guizhou province, China.

https://www.semanticscholar.org/paper/DaylightingAnalysis-of-Vernacular-Architecture-in-XuanWu/7704619e2e73298d381fd44eafa2c1b570492c64.

[25] Aiying, W., Zhuo, L., Dac'y A. (2017). 293: Sustainable daylighting strategy of a Chinese traditional building: FaLun Palace of Yonghe Tibet temple. 25th Conference on Passive and Low Energy Architecture, Dublin.

[26] Duan Z.H., Ford, B.R., Lau, B.E. (2012). The thermal comfort of vernacular skywell dwellings in south-eastern China. Centro de Investigacion de la Arquitectura y la Ciudad; Pontificia Universidad Catolica del Peru (PUCP), Lima, France.

[27] Lin, Z.R., Li, H.Y., Chen, S.H. (2013). Research on simulation of natural lighting of traditional dwellings in Yingtan Village. Huazhong Architecture. 10: 21.

[28] Denan, Z., Majid, N.H., Arifin, N. (2015). Ingenious Malay wood carving as daylight filtering devices. Procedia-Social and Behavioral Sciences, 22(201): 18290. https://doi.org/10.1016/j.sbspro.2015.08.167

[29] Krejcie, R.V., Morgan, D.W. (1970). Determining sample size for research activities. Educational and Psychological Measurement, 30(3): 607-610. https://doi.org/10.1177/001316447003000308 
[30] Williams, A. (2003). How to write and analyse a questionnaire. Journal of Orthodontics, 30(3): 245-252. https://doi.org/10.1093/ortho/30.3.245

[31] Radhakrishna, R.B. (2007). Tips for developing and testing questionnaires/instruments. Journal of Extension, 45(1): $1-4$

[32] Burton, L.J., Mazerolle, S.M. (2011). Survey instrument validity part I: Principles of survey instrument development and validation in athletic training education research. Athletic Training Education Journal, 6(1): $27-$ 35. https://doi.org/10.4085/1947-380X-6.1.27
[33] Abdi, H. (2007). Kendall Rank Correlation. In NJ Salkind (Ed.): Encyclopedia of Measurement and Statistics.

[34] Zysno, P.V. (1997). The modification of the phicoefficient reducing its dependence on the marginal distributions. Methods of Psychological Research Online, 2(1): 41-52.

[35] Greenwood, P.E., Nikulin, M.S. (1996). A guide to chisquared testing (Vol. 280). International Biometric Society, $\quad 54(1)$ : https://doi.org/10.2307/2534027 